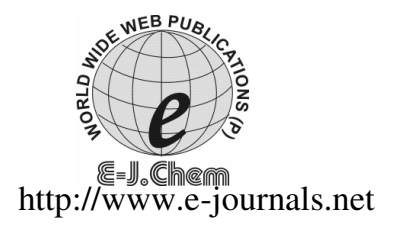

ISSN: 0973-4945; CODEN ECJHAO

E-Journal of Chemistry

2011, 8(4), 1670-1679

\title{
Simultaneous Detemination of Atorvastatin Calcium and Amlodipine Besylate by Spectrophotometry and Multivariate Calibration Methods in Pharmaceutical Formulations
}

\author{
AMIR H. M. SARRAFI*, ELAHE KONOZ and MARYAM GHIYASVAND \\ Department of Chemistry \\ Faculty of Science, Islamic Azad University \\ Central Tehran Branch, P. O. Box 13185-768, Tehran, Iran \\ amirsarrafi45@yahoo.com
}

Received 17 February 2011; Accepted 16 April 2011

\begin{abstract}
Resolution of binary mixture of atorvastatin (ATV) and amlodipine (AML) with minimum sample pretreatment and without analyte separation has been successfully achieved using a rapid method based on partial least square analysis of UV-spectral data. Multivariate calibration modeling procedures, traditional partial least squares (PLS-2), interval partial least squares (iPLS) and synergy partial least squares (siPLS), were applied to select a spectral range that provided the lowest prediction error in comparison to the full-spectrum model. The simultaneous determination of both analytes was possible by PLS processing of sample absorbance between $220-425 \mathrm{~nm}$. The correlation coefficients (R) and root mean squared error of cross validation (RMSECV) for ATV and AML in synthetic mixture were $0.9991,0.9958$ and $0.4538,0.2411$ in best siPLS models respectively. The optimized method has been used for determination of ATV and AML in amostatin commercial tablets. The proposed method are simple, fast, inexpensive and do not need any separation or preparation methods.
\end{abstract}

Keywords: Atorvastatin, Amlodipine, Simultaneous determination, Spectrophotometry, iPLS, siPLS

\section{Introduction}

Amostatin tablets combine the calcium channel blocker amlodipine besylate (3-ethyl-5 methyl ( \pm )-2-[(2-aminoethoxy)methyl]-4-(o-chlorophenyl)-1,4-dihydro-6-methyl 3,5 pyridinedicarboxylate, monobenzene sulphonate) with the lipid-lowering agent atorvastatin calcium $\left(\left[R-\left(\mathrm{R}^{*}, \quad \mathrm{R}^{*}\right)\right] 2\right.$-(4-fluorophenyl)- $\beta, \quad \delta$-dihydroxy-5-(1-methylethyl)-3-phenyl-4[(phenylamino)carbonyl] $1 H$-pyrrole-1-heptanoic acid, calcium salt (2:1) trihydrate). Amostatin tablets are formulated for oral administration in the $20 \mathrm{mg}$ ATV/5 mg AML strength combinations. 
Individual and simultaneous determination with other drugs are possible by spectrophotometry $^{1-5}$, voltammetry ${ }^{6,7}$, HPLC $^{8-12}$, UPLC $^{13}$, HPLC-MS $^{14,15}$, FT-Raman spectrometry $^{16}$, spectrofluoremetry ${ }^{17}$ and HPTLC $^{18}$. In biological samples for individuals and simultaneous determinations with other drugs and metabolites have been reported by: HPLC $^{19-26}$, HPLC-MS ${ }^{15,27-30}$, GC ${ }^{31}$, voltammetry ${ }^{6,7}$ and CZE $^{32}$. The simultaneous determination of ATV and AML by HPLC has been reported ${ }^{33}$.

Multivariate calibration has been described as the most successful application in chemometrics and spectral data combination ${ }^{34}$. In addition, partial least square (PLS) regression is the most popular multivariate calibration technique to build prediction models using spectroscopic signals in many quantitative assays of pharmaceutical formulations ${ }^{35-38}$. Recent applications had been published showing that spectral region selection using suitable algorithms can significantly improve the performance of these full-spectrum calibration techniques $^{39}$. In this case, specific regions are selected generating models with lower prediction error. In practice, multivariate regression models optimization is based on the identification of a complete data subset that will produce the lowest prediction error. An optimized region can be found by reducing or increasing it by subtracting or adding new variables. One of the main advantages of this method is the possibility to represent a regression model in a graphical display, focusing on the better intervals choice and allowing a comparison among interval models and the full-spectrum model. Interval partial least squares (iPLS) allows the building of models with spectral interval and root mean squared error of cross-validation (RMSECV) values can be used as the criterion to evaluate the prediction ability of this interval ${ }^{40}$. However, the exclusion of intervals with higher RMSECV values would lose useful information. This way, advanced models as synergy partial least squares (siPLS) could be applied to find favorable intervals combinations for calibration ${ }^{41}$.

The main objective of this work was to investigate the feasibility of spectrophotometry associated to multivariate linear calibration methods to predicting ATV and AML amount in tablets. Partial least square was employed to data modeling using full spectra information, while iPLS and siPLS were used to select variable intervals.

\section{Experimental}

A JASCO V-530 spectrophotometer with $10 \mathrm{~mm}$ quartz cells was used for measurement of the absorption spectra. All absorption spectra were digitized at one data point per nanometer and transferred in ASCII format to a computer for subsequent analysis and processing by using Matlab 7.1 and Unscrambler 9.1.

\section{Reagents}

Commercial amostatin samples were bought from pharmacies. Analytical grade ATV and AML were obtained from food and drug control laboratories (Tehran, Iran). All other chemical and solvents were of analytical reagent grade.

\section{General procedure}

The stock solutions were prepared daily. For this purpose, appropriate amounts of pure drug were accurately weighed and dissolved with methanol in $50 \mathrm{~mL}$ calibrated flasks, by shaking in an ultrasonic bath for 15 minutes. Aliquots of the stock solutions were added in $25 \mathrm{~mL}$ calibrated flasks, followed by dilution to the mark with the same solvent to obtain the concentrations between the values mentioned in the experimental design part. The absorption spectrum of each sample was recorded between $200-450 \mathrm{~nm}$ at $1 \mathrm{~nm}$ intervals against a blank of methanol. 


\section{Experimental design}

Calibration and test sets for two component systems were designed according to factorial principles. Solutions containing drug concentrations in the range $0.0-32.0 \mu \mathrm{g} \mathrm{mL}^{-1}$ for ATV and $0.0-8.0 \mu \mathrm{g} \mathrm{mL}^{-1}$ for AML were produced by dilution of the stock solutions. A five level factorial design was used to produce a full set of 25 samples. A three level set was derived from this to produce a calibration set of nine samples, with the remaining 16 samples used for an independent test set.

\section{Pharmaceutical sample preparation}

After grinding and homogenizing of an accurately weighted of 20 tablets, a weighted sample equal to mean weight of one tablet was used for analysis. Each weighed sample was mixed with $70 \mathrm{~mL}$ methanol and the mixture was subjected to ultrasonication for 15 minutes. After cooling and further dilution to $100 \mathrm{~mL}$ with methanol, a $10 \mathrm{~mL}$ portion of the sample was centrifuged at $3000 \mathrm{rev} / \mathrm{min}$. $2 \mathrm{~mL}$ portion of the supernatant was then diluted to $25 \mathrm{~mL}$ with methanol. The absorption spectra between 200 and $400 \mathrm{~nm}$ against methanol was recorded for all solutions. Spiking experiments using standard addition method checked reliabilities.

\section{Chemometrics methods}

Multivariate chemometrics methods were applied to obtain quantitative information from the measurements. Partial least square regression methods were applied to spectral data to built calibration models enabling prediction of ATV and AML amount in pharmaceutical preparations. The root mean squared error of full cross-validation (RMSECV) and root mean squared error of prediction (RMSEP) were used to select the number of latent variables and to evaluate the prediction ability between different PLS models. The RMSECV calculated according to the following equation:

$$
R M S E C V=\sqrt{\left(\left(\sum_{i=1}^{n} y i-y \tilde{i}\right)^{2} / n\right)}
$$

Where $y \tilde{i}$ s the predicted value for sample $i, y i$ the reference value for sample $i$ and $n$ is the number of observations. The RMSEP calculated according to the following equation:

$$
R M S E P=\sqrt{\left(\left(\sum_{i-1}^{m}(z i-z \tilde{i})^{2}\right) / m\right)}
$$

Where $z \tilde{i}$ is the predicted value for test set sample $i, z i$ the reference value for test set sample $i$ and $m$ is the number of observations in testing set.

\section{Results and Discussion}

\section{Spectral behavior}

In Figure 1, the absorption spectra of ATV, AML, synthetic mixture and tablet solutions in methanol recorded between 220 and $425 \mathrm{~nm}$ are shown. The stability of ATV, AML and commercial sample solutions were checked for $4 \mathrm{~h}$ and the UV-Vis absorption spectra of all sample solutions were found to be stable for this period of time. It is also to be noted that the simultaneous determination of the aforementioned drugs with the proposed method can be carried out in less than $1 \mathrm{~h}$.

\section{Full spectrum PLS model}

In order to have a measurement of the variable selection algorithms quality, models were built using UV-Vis full spectrum information. Full spectrum PLS models were obtained with two latent variables for ATV and AML and results are shown in Tables 1 and 2. 

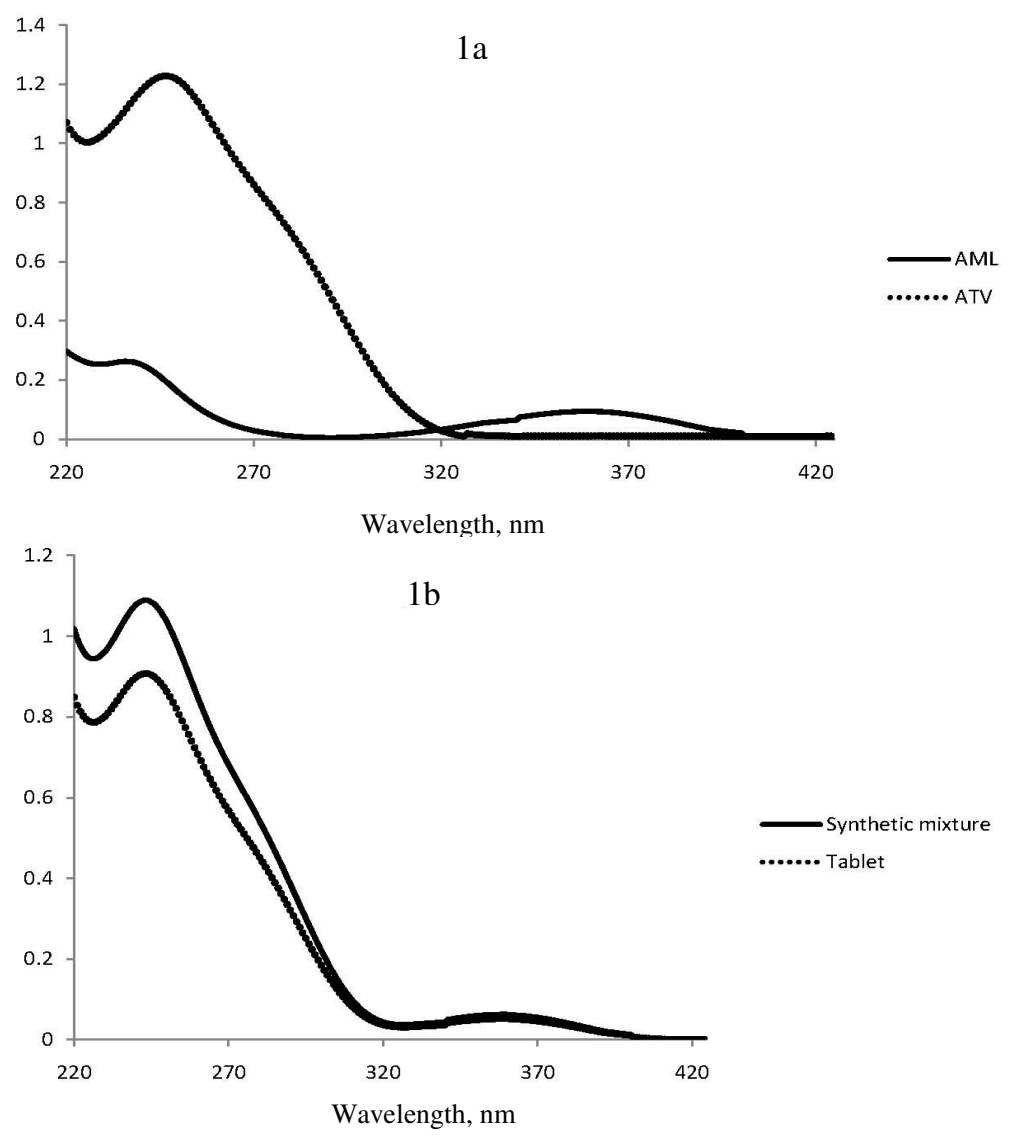

Figure 1. The absorption spectra of ATV and AML (1a), synthetic mixture and tablet (1b) solutions in methanol

Table 1. Statistical results to ATV better calibration models

\begin{tabular}{cccccccc}
\hline Model & $\mathrm{VN}^{\mathrm{a}}$ & Intervals & $\mathrm{LV}^{\mathrm{b}}$ & $\mathrm{RMSECV} \mu \mathrm{g} \mathrm{\textrm {mL } ^ { - 1 }}$ & $\mathrm{R}$ & RMSEP, $\mu \mathrm{g} \mathrm{mL}^{-1}$ & $\mathrm{R}$ \\
\hline PLS & 206 & All & 2 & 0.4901 & 0.9989 & 0.5577 & 0.9986 \\
PLS-2 & 125 & - & 2 & 0.4852 & 0.9989 & 0.5509 & 0.9986 \\
iPLS5 & 41 & 2 & 2 & 0.4581 & 0.9991 & 0.5168 & 0.9986 \\
iPLS10 & 21 & 5 & 2 & 0.4589 & 0.9991 & 0.5372 & 0.9985 \\
iPLS15 & 14 & 5 & 2 & 0.4540 & 0.9991 & 0.5173 & 0.9985 \\
siPLS5 & 82 & 2,4 & 2 & 0.4776 & 0.9990 & 0.5388 & 0.9986 \\
siPLS5 & 123 & $2,3,4$ & 2 & 0.4780 & 0.9990 & 0.5384 & 0.9986 \\
siPLS10 & 41 & 5,10 & 2 & 0.4576 & 0.9991 & 0.5336 & 0.9985 \\
siPLS10 & 62 & $3,4,10$ & 2 & 0.4596 & 0.9990 & 0.6206 & 0.9981 \\
siPLS10 & 82 & $4,5,7,10$ & 2 & 0.4603 & 0.9990 & 0.5584 & 0.9983 \\
siPLS15 & 28 & 4,6 & 2 & 0.4538 & 0.9991 & 0.5122 & 0.9986 \\
siPLS15 & 42 & $6,7,10$ & 2 & 0.4538 & 0.9991 & 0.5122 & 0.9986 \\
siPLS15 & 55 & $6,7,10,15$ & 2 & 0.4538 & 0.9991 & 0.5122 & 0.9986 \\
\hline
\end{tabular}

${ }^{a}$ VN: total variables numbers, ${ }^{b}$ LV: latent variables numbers 
Table 2. Statistical results to AML better calibration models

\begin{tabular}{cccccccc}
\hline Model & $\mathrm{VN}^{\mathrm{a}}$ & Intervals & $\mathrm{LV}^{\mathrm{b}}$ & $\mathrm{RMSECV}, \mu \mathrm{g} \mathrm{\textrm {m } ^ { - 1 }}$ & $\mathrm{R}$ & $\mathrm{RMSEP} \mu \mathrm{g} \mathrm{mL}^{-1}$ & $\mathrm{R}$ \\
\hline PLS & 206 & All & 2 & 0.3338 & 0.9919 & 0.4406 & 0.9955 \\
PLS-2 & 125 & - & 2 & 0.3104 & 0.9874 & 0.3903 & 0.9958 \\
iPLS5 & 41 & 3 & 3 & 0.2534 & 0.9953 & 0.3936 & 0.9952 \\
iPLS10 & 20 & 8 & 2 & 0.2708 & 0.9947 & 0.5023 & 0.9950 \\
iPLS15 & 14 & 8 & 3 & 0.2635 & 0.9950 & 0.4298 & 0.9946 \\
siPLS5 & 82 & 3,5 & 3 & 0.2547 & 0.9953 & 0.4505 & 0.9956 \\
siPLS5 & 123 & $3,4,5$ & 4 & 0.2611 & 0.9951 & 0.4588 & 0.9960 \\
siPLS10 & 42 & 5,6 & 3 & 0.2546 & 0.9953 & 0.3950 & 0.9953 \\
siPLS10 & 62 & $5,6,10$ & 3 & 0.2540 & 0.9953 & 0.3948 & 0.9953 \\
siPLS10 & 82 & $5,6,9,10$ & 3 & 0.2546 & 0.9953 & 0.4339 & 0.9956 \\
siPLS15 & 28 & 3,8 & 5 & 0.2411 & 0.9958 & 0.2213 & 0.9973 \\
siPLS15 & 41 & $3,8,15$ & 3 & 0.3240 & 0.9924 & 0.5368 & 0.9931 \\
siPLS15 & 54 & $2,11,12,14$ & 2 & 0.3420 & 0.9915 & 0.4844 & 0.9958 \\
\hline
\end{tabular}

\section{PLS-2 model}

${ }^{a}$ VN: total variables numbers., ${ }^{b}$ LV: latent variables numbers

To select the spectral region and optimum latent variable numbers, modeling according to previous works in different spectral region doing repeatedly and the spectral region that lead to the lowest values of RMSECV and RMSEP was selected ${ }^{35,36}$. The spectral region between 236 and $360 \mathrm{~nm}$ was selected for analysis and as a consequence, 125 experimental points per spectrum were used. Table 1 and 2 show the statistical indicators to better PLS- 2 calibration model.

\section{ATV iPLS models}

The iPLS models were built on spectra division into 5, 10 and 15 intervals. The iPLS routine generates graphical information indicating the optimum number of latent variables used in each interval model, RMSECV and RMSEP values. In this case, the subinterval that presented the minor RMSECV and RMSEP values was selected. The central iPLS plots, the RMSECV values for each interval selected (bars) and RMSECV values to full spectrum model (dotted line) using two latent variables are demonstrated in Figure 2. Table 1 shows the statistical indicators to better ATV iPLS calibration models developed with 5, 10 and 15 intervals. The developed model using the interval 5 for iPLS with 15 intervals produced better results according to lower results for RMSECV and RMSEP values. This model using 2 latent variables and only 14 variable numbers and the selected interval included the $276-290 \mathrm{~nm}$ region.

\section{ATV siPLS modesl}

The siPLS algorithm principle is to split the data set into a number of intervals and to calculate all possible PLS model combinations of two, three or four intervals. Thereafter, the combined subinterval models is compared with the other models. For the developed PLS models using spectra full information, the including uninformative wavelengths could negatively affect the calibration by producing both large relative bias. In this case, a judicious selection of spectral regions would improve the predictive ability of the PLS model. Therefore, variables selection by siPLS was implanted to verify if the combination of more than one interval would result in models with better predictive ability. The spectrum was divided in 5,10 or 15 intervals combined in up to 4 subintervals. The spectrum divided in 5 intervals was combined in up to 3 subintervals and the spectrum divided in 10 and 15 intervals was combined in up to 5 intervals. Table 1 shows the statistical indicator to ATV 
siPLS better calibration models. All models using the spectrum subdivided in 15 intervals, after the variable selection by siPLS, showed lower RMSECV and RMSEP. The siPLS model with the intervals number 4 and 6 is the better model because of using lower variable numbers. This model using 2 latent variables and 28 variable numbers and the selected intervals included the 262-276 nm (interval 4) and 290-304 nm (interval 6). For this siPLS model, the results showed a good correlation between reference and predicted values, indicated by a correlation coefficient of 0.9991 , as shown in Figure 3.

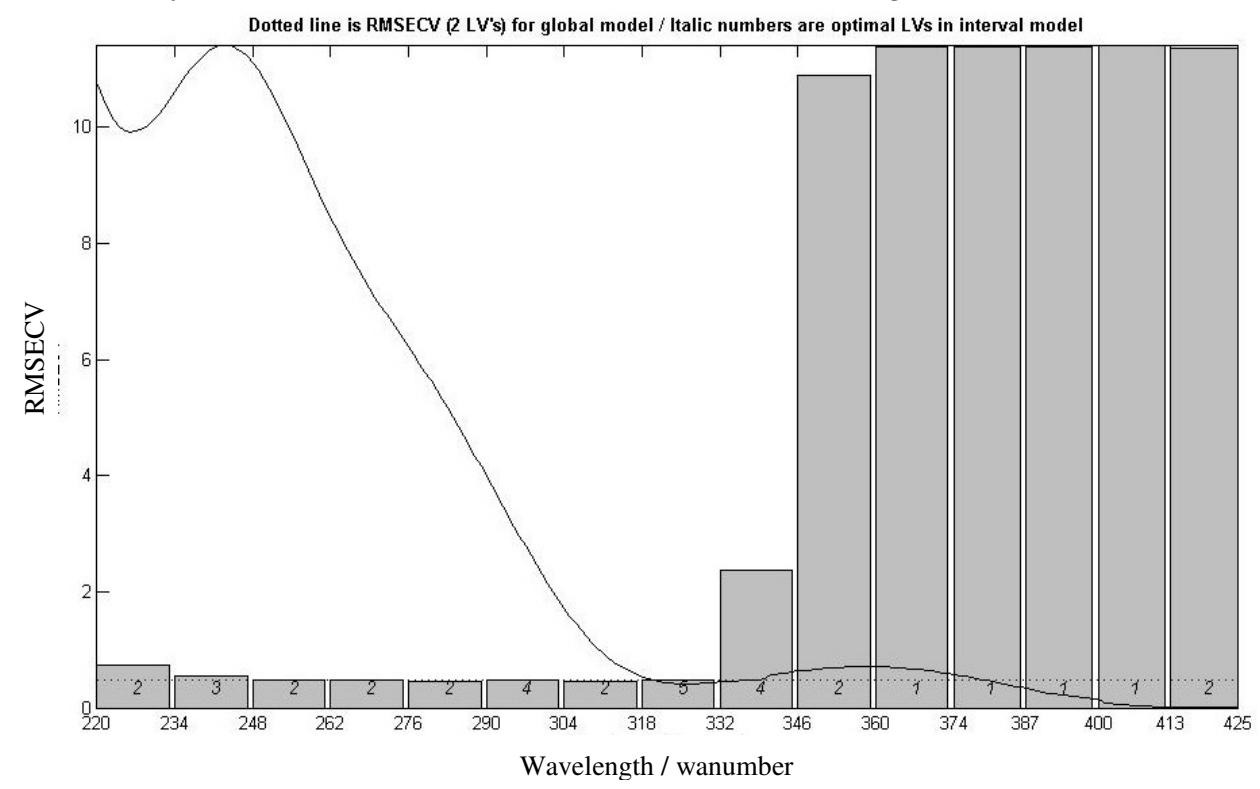

Figure 2. Cross-validated prediction errors (RMSECV) values to full-spectrum model and interval models (bars) to ATV determination using PLS and iPLS algorithms (dotted line and numbers above interval numbers refer to full-spectrum RMSECV and latent variables using in each model, respectively)

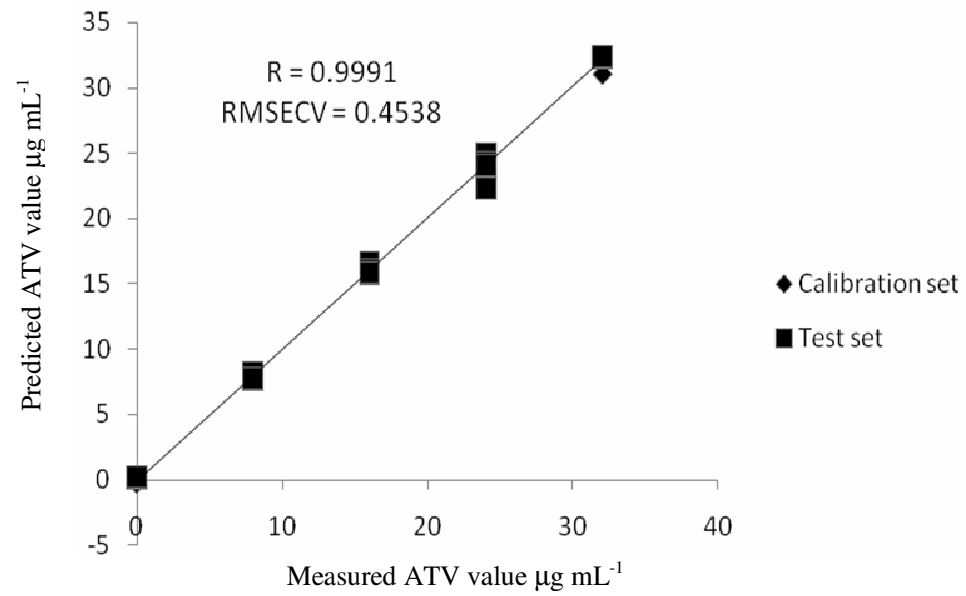

Figure 3. Predicted values versus measured ATV values for siPLS model, using intervals 4 and 6 and 2 latent variables 


\section{AML iPLS models}

Figure 4 shows the central iPLS plots, the RMSECV values for each interval selected (bars) and RMSECV values to full spectrum model (dotted line) using two latent variables. Table 2 shows the statistical indicator to AML iPLS calibration models using the spectrum subdivided in 5, 10 and 15 intervals. The developed model using the interval 3 for iPLS with 5 intervals produced better results according to lower results for RMSECV and RMSEP values. This model using 3 latent variables and only 41 variable numbers and the selected interval included the 303-344 $\mathrm{nm}$ region.

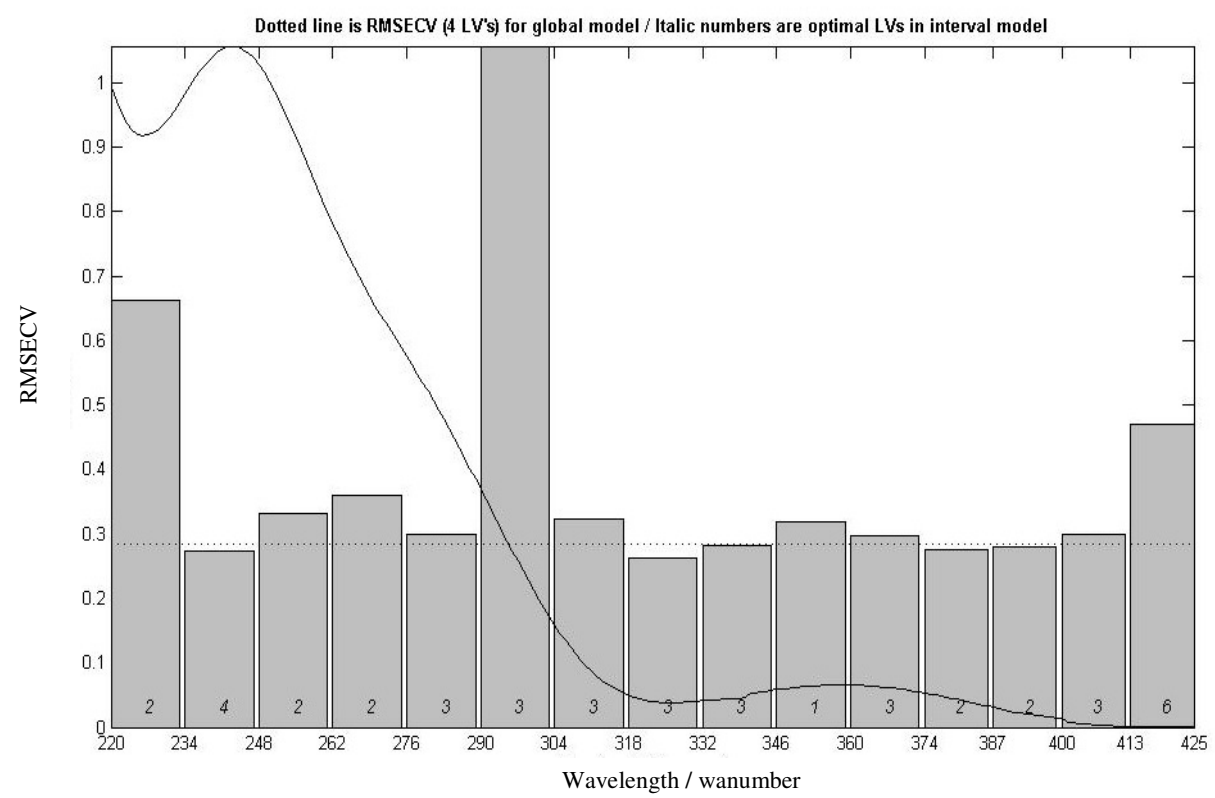

Figure 4. Cross-validated prediction errors (RMSECV) values to full-spectrum model and interval models (bars) to AML determination using PLS and iPLS algorithms (dotted line and numbers above interval numbers refer to full-spectrum RMSECV and latent variables using in each model, respectively)

\section{AML siPLS models}

The algorithm siPLS was implanted using the spectrum subdivided in 5, 10 or 15 intervals combined in up to 3, 4 and 4 subintervals respectively. Table 2 shows the statistical indicators to AML siPLS calibration models. The siPLS model using the spectrum subdivided in 15 intervals with the intervals number 3 and 8 is the better model according to lower results for RMSECV and RMSEP values. This model using 5 latent variables and 28 variable numbers and the selected intervals included the $248-262 \mathrm{~nm}$ (interval 3 ) and 318-332 nm (interval 8). For this siPLS model, the results showed a good correlation between reference and predicted values, indicated by a correlation coefficient of 0.9958 , as shown in Figure 5.

\section{Comparision of PLS models}

Comparing the results from full PLS, PLS-2, iPLS and siPLS models in determination of pharmaceuticals by spectrophotometry, siPLS models showed better predictive capacity (lower RMSEP). This result could be explained by three reasons: (1) full PLS models used 
all spectral information therefore included too many variable numbers; (2) PLS-2 model used selected spectral region that models two components simultaneously, but have many variable numbers. (3) iPLS models can reduce variables by selecting specific spectral regions, however useful spectral information can be lost; (4) with intervals combination performed by siPLS obtaining models with reduced total variables numbers and better predictive capacity (without information loosing) will be possible.

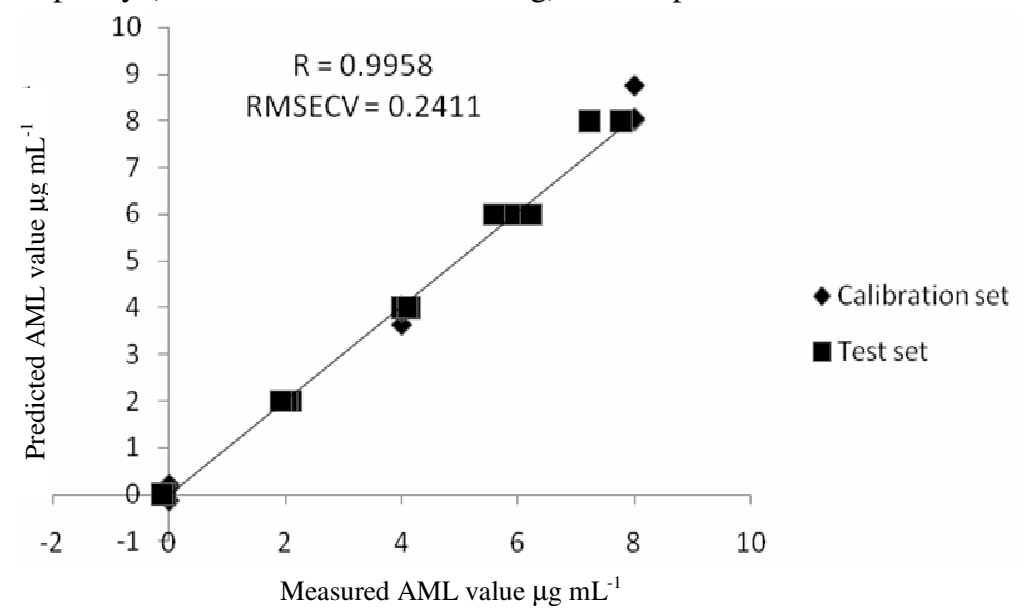

Figure 5. Predicted values versus measured AML values for siPLS model, using intervals 3 and 8 and 5 latent variables

\section{Application of siPLS better methods}

Commercial tablets products from Alhavi and Razak companies were analyzed. Spiking experiments using standard addition method checked reliabilities. The percent of recoveries and relative standard deviation for each element in spiked samples are given in Table 3 and 4. As can be seen, satisfactory results were obtained in all cases by the siPLS proposed methods. Limits of detection (LOD's) were calculated as three standard error of estimation (SEE) values ${ }^{35,36}$. LOD's of $0.7104 \mu \mathrm{g} \mathrm{mL}^{-1}$ for AML, $1.0704 \mu \mathrm{g} \mathrm{mL}^{-1}$ for ATV were obtained in siPLS models, respectively.

Table 3. Analyses of amostatin tablet from Razak Company

\begin{tabular}{cccccc}
\hline Analyte & $\begin{array}{c}\text { Added, } \\
\text { mg per tablet }\end{array}$ & $\begin{array}{c}\text { Found, } \\
\text { mg per tablet }\end{array}$ & $\%$ RSD $^{\mathrm{a}}$ & $\begin{array}{c}\% \\
\text { Recovery }\end{array}$ & $\begin{array}{c}\text { Declared content, } \\
\text { mg per tablet }\end{array}$ \\
\hline ATV & 0.00 & 19.35 & 1.20 & - & 20 \\
AML & 0.00 & 4.93 & 1.10 & - & 5 \\
\hline ATV & 10.00 & 29.45 & 1.00 & 101.0 & - \\
AML & 2.50 & 7.46 & 1.21 & 101.2 & - \\
\hline ATV & 15.00 & 34.55 & 1.15 & 101.3 & - \\
AML & 3.75 & 8.70 & 1.10 & 100.5 & - \\
\hline ATV & 20.00 & 39.25 & 1.18 & 99.5 & - \\
AML & 5.00 & 9.90 & 1.05 & 99.4 & - \\
\hline
\end{tabular}

${ }^{a}$ Relative standard deviation for $n=3$ 
Table 4. Analyses of amostatin tablet from Alhavi Company

\begin{tabular}{cccccc}
\hline Analyte & $\begin{array}{c}\text { Added, } \\
\text { mg per tablet }\end{array}$ & $\begin{array}{c}\text { Found, } \\
\text { mg per tablet }\end{array}$ & $\%$ RSD $^{\mathrm{a}}$ & $\begin{array}{c}\% \\
\text { Recovery }\end{array}$ & $\begin{array}{c}\text { Declared content, } \\
\text { mg per tablet }\end{array}$ \\
\hline ATV & 0.00 & 19.20 & 1.10 & - & 20 \\
AML & 0.00 & 4.90 & 1.00 & - & 5 \\
\hline ATV & 10.00 & 28.95 & 1.17 & 96.80 & - \\
AML & 2.50 & 7.35 & 1.11 & 98.00 & - \\
\hline ATV & 15.00 & 34.54 & 1.21 & 102.3 & - \\
AML & 3.75 & 8.60 & 1.20 & 98.70 & - \\
\hline ATV & 20.00 & 39.00 & 1.22 & 99.0 & - \\
AML & 5.00 & 9.90 & 1.12 & 100.0 & - \\
\hline
\end{tabular}

\section{Conclusion}

${ }^{a}$ Relative standard deviation for $n=3$

Using the PLS regression algorithm combined with spectrophotometry data it was possible to develop multivariate models for simultaneous determination of ATV and AML in commercial pharmaceutical products. Spiked assay results, expressed as the percentage of recovery were found to be $96.8-102.3 \%$ for ATV and 98.0-101.2\% for AML. The siPLS variable selection techniques used in this work produced models with better predictive ability compared to other PLS models. The siPLS algorithm demonstrated to be more suitable in the combined regions selection having relevant information. The proposed method is simple and allows potential applications to simultaneous, fast and reliable determination of ATV and AML in solid pharmaceutical dosage forms.

\section{References}

1. Rahman N and Azmi S N H, Il Farmaco, 2001, 56, 731.

2. Rahman N and Hoda M N, J Pharm Biomed Anal., 2003, 31, 381.

3. Basavaiah K, Chandrashekar U and Prameela H C, Il Farmaco, 2003, 58, 141.

4. $\quad$ Rahman N, Singh M and Hoda M N, Il Farmaco, 2004, 59(11), 913-919.

5. Shama S A, Amin A S, Mabrouk E M and Omara H A, Arab J Chem., 2009, 2, 59-63.

6. Gazy A A K, Talanta, 2004, 62, 575.

7. Goyal R N and Bishnoi S, Bioelectrochem., 2010, 79(2), 234-240.

8. Argekar A P and Powar S G, J Pharm Biomed Anal., 2000, 21, 1137-1142.

9. Erturk S, Akta E S, Ersoy L and Ficiciolu S, J Pharm Biomed Anal., 2003, 33, 1017.

10. Naidu K R, Kale U N and Shingare M S, J Pharm Biomed Anal., 2005, 39, 147-155.

11. Novakova L, Satinsky D and Solich P, TrAC Trend Anal Chem., 2008, 27, 352-367.

12. Dongre V G, Shah S B Shah, Karmuse P P, Phadke M and Jadhav V K, J Pharm Biomed Anal., 2008, 46(3), 583-586.

13. Kadav A A and Vora D N, J Pharm Biomed Anal., 2008, 48, 120-126.

14. Zhong D, Chen X, Gu J, Li X and Guo J, Clin Chim Acta, 2001, 313(1), 147-150.

15. Miao X and Metcalfe C D, J Chromatogr A, 2003, 998, 133-141.

16. Skorda D and Kontoyannis C G, Talanta, 2008, 74, 1066-1070.

17. Abdel-Wadood H M, Mohamed N A and Mahmoud A M, Spectrochim Acta Part A, 2008, 70(3), 564-570.

18. Pandya K K, Milan S, Gandhi T P, Modi I A Modi, Modi R I and Chakravarthy B K, J Chromatogr B, 1995, 667(2), 315-320.

19. Josefsson M, Zackrisson A and Norlander B, J Chromatogr B, 1995, 672(2), 310-313.

20. Josefsson M and Norlander B, J Pharm Biomed Anal., 1996, 15, 267-277. 
21. Tatar S and Atmaca S, J Chromatog, B, 2001, 758(2), 305-310.

22. Bahrami G and Mirzaeei S H, J Pharm Biomed Anal., 2004, 36(1), 163-168.

23. Bahrami G, Mohammadi B, Mirzaeei S and Kiani A, J Chromatogr B, 2005, 826, 41-45.

24. Zarghi A, Foroutan S M, Shafaati A and Khoddam A, Il Farmaco, 2005, 60(9), 789-792.

25. Farahani H, Norouzi P, Beheshti A, Sobhi H R, Dinarvand R and Ganjali M R, Talanta, 2009, 80(2), 1001-1006.

26. Wei X, Yang G, Qi L and Chen Y, Talanta, 2009, 77(3), 1197-1202.

27. Streel B, Laine C, Zimmer C, Sibenaler R and Ceccato A, J Biochem Bioph Meth., 2002, 54, 357-368.

28. Kristoffersen L, Oiestad E L, Opdal M S, Krogh M, Lundanes E and Christophersen A S, J Chromatogr B, 2007, 850, 147.

29. Ma Y, Qin F, Sun X, Lu X and Li F, J Pharm Biomed Anal, 2007, 43(4), 1540-1545.

30. Sarkar A K, Ghosh D, Das A, Selvan P S, Gowda K V, Mandal U, Bose A, Agarwal S, Bhaumik U and Pal T K, J Chromatogr B, 2008, 873, 77-85.

31. Monkman S C, Ellis J S, Cholerton S, Thomason J M, Seymour R A and Idle J R, $J$ Chromatogr B, 1996, 678(2), 360-364.

32. Mikus P, Marakova K, Marak J, Nemec I, Valaskova I and Havranek E, $J$ Chromatogr B, 2008, 875, 266-278.

33. Mohammadi A, Rezanour N, Ansari Dogaheh M, Ghorbani Bidkorbeh F, Hashem M and Walker R B, J Chromatogr B, 2007, 846, 215-221.

34. Brereton R G, Analyst, 2000, 125, 2125-2154.

35. Sarrafi A H M, Khodakarami Z and Karkeabadi M, E-J Chem., 2009, 6, S111-S116.

36. Sarrafi A H M, Konoz E and Feyzbakhsh A, E-J Chem., 2010, 7(3), 997-1002.

37. Soto C, Contreras D, Orellana S, Yanez J and Torel M I, Anal Sci., 2010, 26(8), 891-896

38. Mot A, Soponar F, Medvedovici A and Sarbu C, Anal Lett., 2010, 43(4-6), 804-813.

39. Norgaard L, Hahn M T, Knudsen L B, Farhat I A and Engelsen S B, Int Dairy J., 2005, 15(12), 1261-1270.

40. Pereira A F C, Pontes M J C, Neto F F G, Santos S R B, Galvao R K H and Araujo M C U, Food Res Int., 2008, 41, 341-348.

41. Chen Q, Zhao J, Liu M, Cai J and Liu J, J Pharm Biomed Anal., 2008, 46(3), 568-573. 


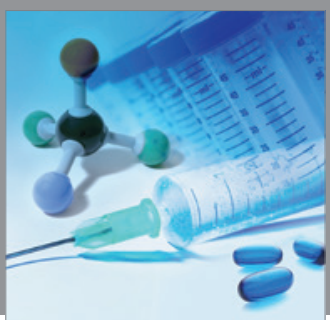

International Journal of

Medicinal Chemistry

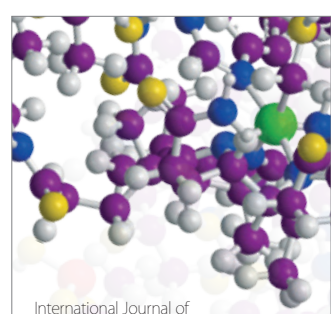

Carbohydrate Chemistry

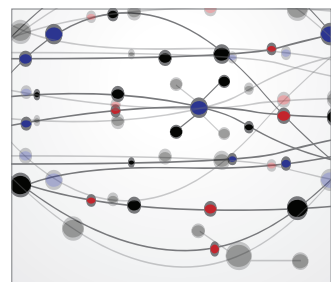

The Scientific World Journal
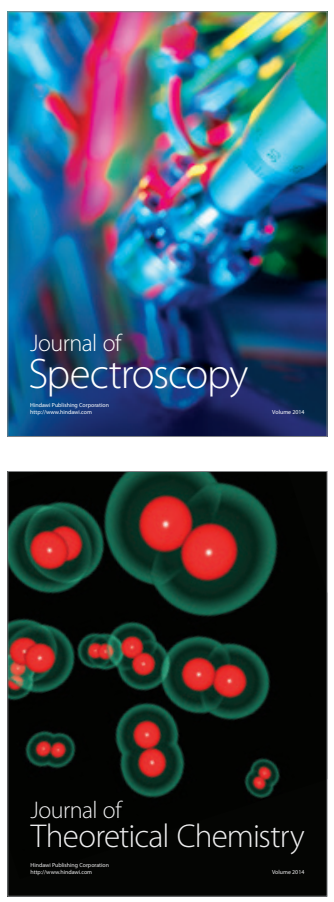
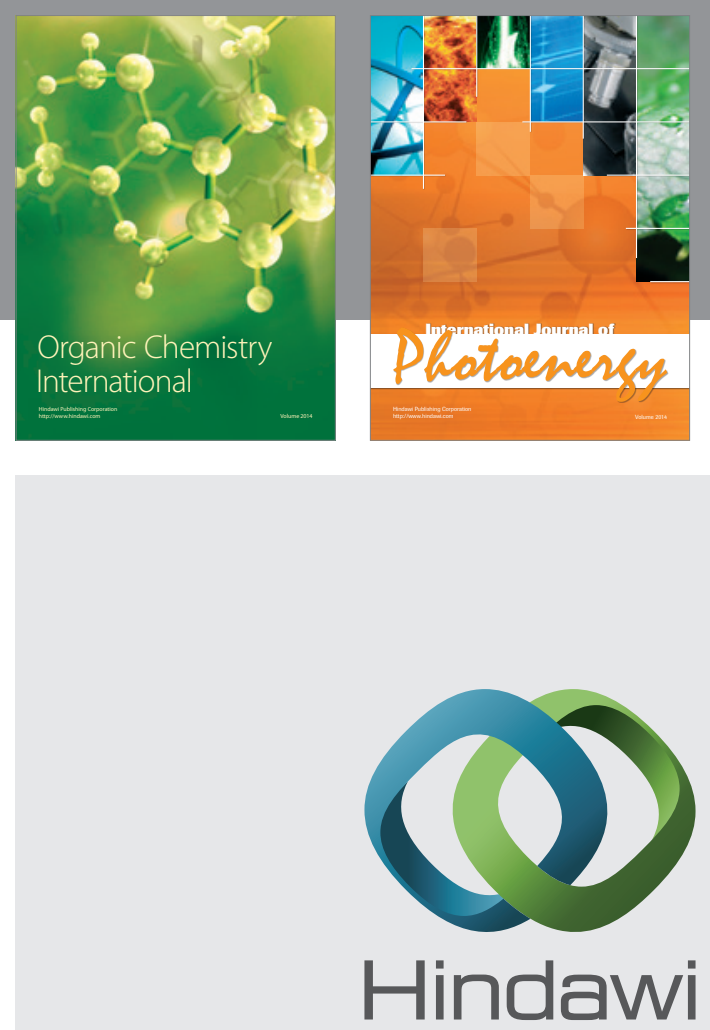

Submit your manuscripts at

http://www.hindawi.com
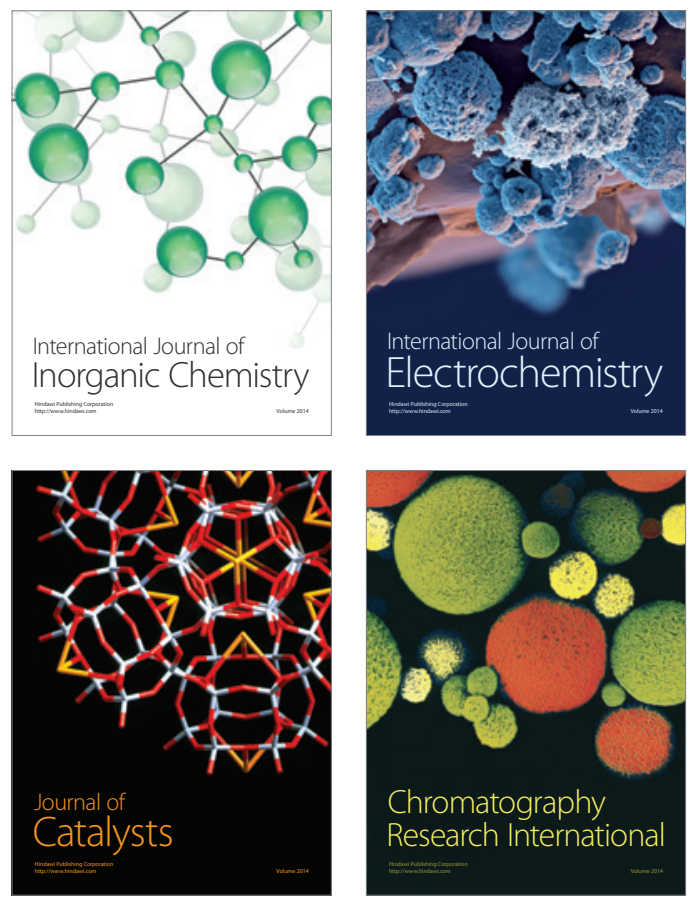
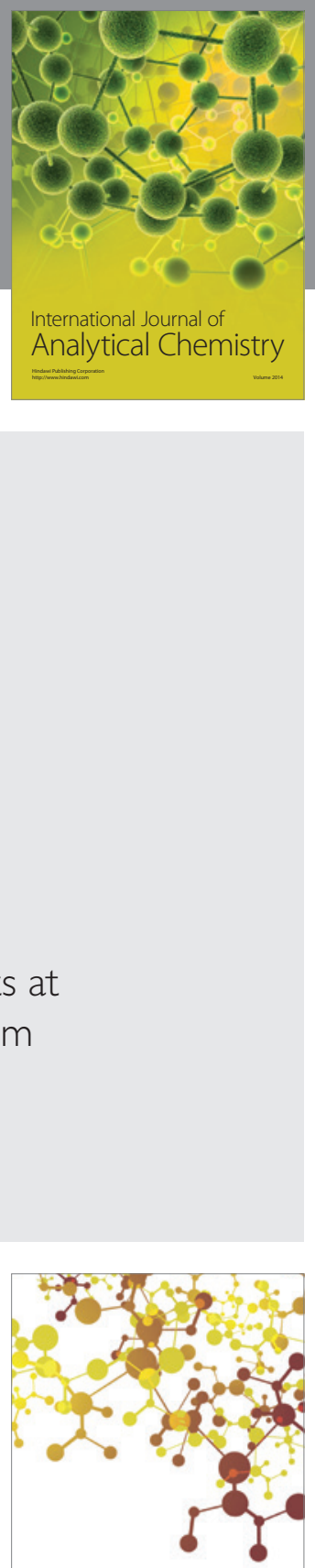

Journal of

Applied Chemistry
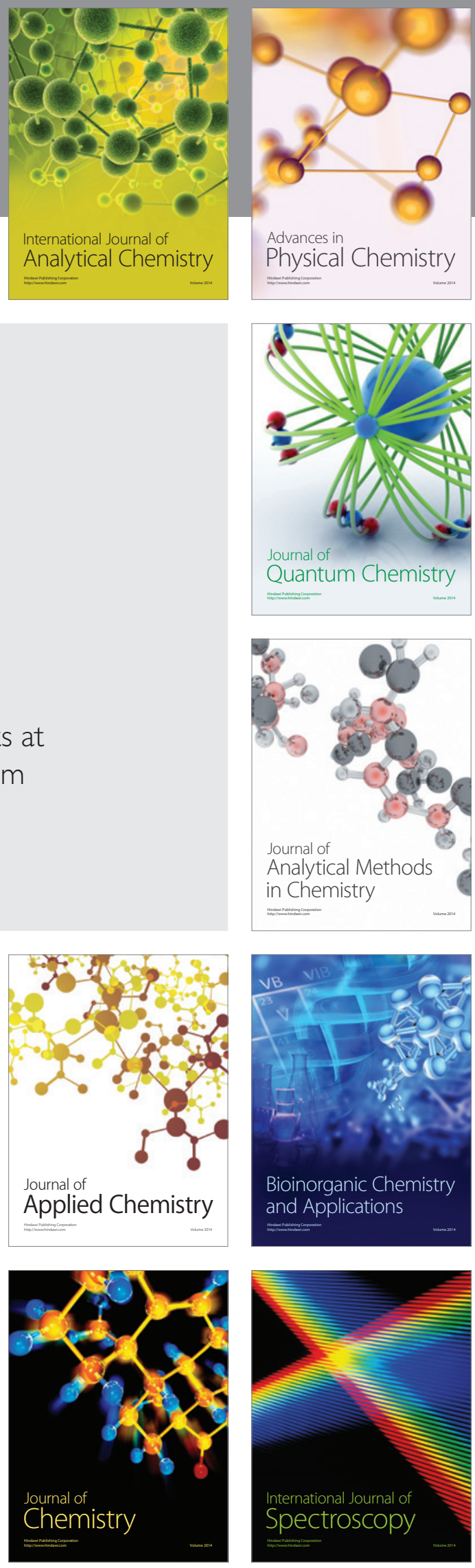